\title{
Lidil
}

Revue de linguistique et de didactique des langues

$56 \mid 2017$

Grammaires et littéracies

\section{Outils linguistiques et entrée en littéracie chez les élèves allophones}

Linguistics Tools for Allophone Pupils Entering Literacy

Fatima Chnane-Davin et Jean-Pierre Cuq

\section{(2) OpenEdition}

1 Journals

Édition électronique

URL : http://journals.openedition.org/lidil/4726

DOI : $10.4000 /$ lidil. 4726

ISSN : 1960-6052

Éditeur

UGA Éditions/Université Grenoble Alpes

Édition imprimée

ISBN : 978-2-37747-024-2

ISSN : $1146-6480$

Référence électronique

Fatima Chnane-Davin et Jean-Pierre Cuq, « Outils linguistiques et entrée en littéracie chez les élèves allophones », Lidil [En ligne], 56 | 2017, mis en ligne le 01 novembre 2017, consulté le 21 décembre 2020. URL : http://journals.openedition.org/lidil/4726 ; DOI : https://doi.org/10.4000/lidil.4726

Ce document a été généré automatiquement le 21 décembre 2020.

(C) Lidil 


\title{
Outils linguistiques et entrée en littéracie chez les élèves allophones
}

\author{
Linguistics Tools for Allophone Pupils Entering Literacy
}

Fatima Chnane-Davin et Jean-Pierre Cuq

\section{Introduction}

1 On s'intéressera ici à l'entrée dans la littéracie des élèves allophones ayant le français comme langue seconde en France. À l'inverse de l'oral, l'écrit est souvent perçu par ces élèves comme un monde de communication non ou peu fonctionnel, et en tout cas moins urgent que l'oral qui s'acquiert à la fois dans et hors de l'école. Pourtant, dans un monde imprégné d'écrit, ordinaire ou académique, sa maitrise est un objectif prioritaire que l'école, avec plus ou moins de succès, poursuit sans relâche parce que c'est sa mission première et qu'elle est le seul lieu possible de son enseignement à des jeunes. Plus que pour d'autres souvent, l'entrée de ces élèves dans la littéracie présente des difficultés qui se compliquent encore par l'exigence de rapidité d'apprentissage à laquelle ils sont soumis. Cette réduction de l'espace et du temps d'appropriation (Cuq, 2014) amène à une réduction formalisée de l'objet d'apprentissage, perçue comme une étape nécessaire à une utilisation efficace dans la vie scolaire et extrascolaire.

Le recours à la grammaire est l'outil majeur de l'organisation de cette étape d'entrée dans la littéracie et des outils métalinguistiques de description de la langue sont mis en œuvre pour aider à la compréhension des textes au cours d'activités orales comme le questionnement ou la formulation d'hypothèse. On table sur le fait que leur intégration dans la boite à outil mentale des élèves permettra la production de textes sémantiquement et formellement structurés. Au début au moins, l'élève ne ressent pas le besoin de connaitre ces outils, mais sait très vite qu'on attend de lui qu'il sache nommer et reconnaitre les constituants de la phrase, du texte et du discours. Il rendra ces outils d'autant plus opérationnels en termes de compréhension et de production, qu'il comprendra mieux l'enjeu de leur mise en œuvre en classe (Cuq, 1996; Vigner, 2004 ; Véronique, 2009). 
3 Notre étude se fondera sur deux corpus. Avec le premier, issu d'une séance de classe de $4^{\mathrm{e}}$ FLS $^{1}$ enregistrée dans un collège REP ${ }^{2}$ où l'enseignant travaille sur une " étude de texte ", nous verrons comment les outils grammaticaux aident à l'entrée en littéracie en lecture par la construction de la compréhension fine d'un texte. Avec le second, issu d'une recherche longitudinale de productions écrites d'élèves allophones nouvellement arrivés (EANA), nous examinerons le réinvestissement en écriture de ces outils linguistiques.

\section{Littéracie et grammaire}

4 Nous ne discuterons pas des multiples définitions proposées depuis un quart de siècle maintenant pour la littéracie des deux côtés de l'Atlantique. Il en est suffisamment fait écho dans ce numéro pour que nous n'ayons pas à entrer dans le débat théorique, avec plus ou moins de conviction, mais nous devons dire d'entrée de jeu le sens que nous donnerons à ce terme dans le présent article, sans préjuger d'une autre acception dans d'autres cas, voire d'une discussion plus radicale sur la validité du concept de littéracie en tant que concept didactique (Chiss, 2008). Il s'agit d'un sens assez restreint, celui de l'essai d'amélioration de la compétence de communication écrite. De façon classique, celle-ci se décline en termes de lecture (savoir lire, au sens complet du terme, c'est-à-dire être capable de mettre un sens non seulement derrière les mots, mais derrière les textes) et de production écrite, c'est-à-dire être capable de produire des textes structurés et porteurs de sens.

5 La souplesse que nous voyons dans le concept de littéracie est un argument assez commode pour nous permettre cette restriction qui n'envisage pas les éventuels effets de retour de l'écrit sur l'amélioration de la compétence communicative orale. L'acquisition qu'on pourrait dire littéraciée d'une expression orale suffisamment différenciée de l'oral ordinaire pour être acceptable et acceptée comme un oral scolaire n'entrera donc pas dans notre propos. En revanche, nous verrons tout au long de l'étude de notre corpus que l'interaction orale des élèves entre eux et du maitre avec les élèves est une condition nécessaire à la bonne poursuite des objectifs écrits, tant en compréhension qu'en production.

6 L'argument qu'on avancera surtout pour notre choix restrictif est celui de la contextualisation de notre étude. Il s'agit d'une situation de langue seconde en contexte scolaire français. Dans ce contexte particulier, les élèves concernés ne doivent pas seulement acquérir, si ce n'est déjà fait ou partiellement fait à leur arrivée à l'école, un savoir-faire procédural de base en termes de communication orale et écrite, ils doivent amener ce savoir-faire à un niveau compatible à leur insertion dans une classe ordinaire. C'est ce que le Socle commun de connaissances, de compétences et de culture appelle maitrise de la langue.

7 Cette insertion dans une classe ordinaire suppose aussi de la part des élèves la possession d'un certain savoir déclaratif, notamment dans le domaine grammatical. Contrairement peut-être à d'autres traditions scolaires, l'importance accordée en France à ce savoir déclaratif reste assez grande, même si elle a pu être sujette à des variations tant en termes quantitatifs que qualitatifs, ou même d'options théoriques, dont nous ne discuterons pas non plus ici de la validité. 
8 L'objectif des enseignants des classes de français langue seconde étant de faire le plus rapidement possible des élèves nouvellement arrivés des élèves ordinaires, ils se voient donc confrontés à une double difficulté : choisir une option méthodologique qui optimise le savoir-faire linguistique de ces élèves, notamment à l'écrit, et en même temps, leur faire acquérir un minimum de connaissances déclaratives.

9 La question de la transférabilité ou non de ce savoir en savoir-faire n'est pas de leur ressort pour deux raisons. La première est culturelle : faire apprendre la grammaire aux enfants et aux jeunes est plus ou moins consciemment conçu dans notre société comme la nécessaire transmission d'un savoir patrimonial et social, dont la valeur éducative n'est certes pas démontrée (Cuq, 2002) mais dont la valeur identitaire est encore très forte. Il s'agit donc d'une tradition scolaire, d'une sorte d'habitus collectif si on veut. La seconde, qui découle de la première est que la transférabilité est considérée comme un fait, non sujet à discussion, par l'autorité éducative. Celle-ci raisonne sur le cas du plus grand nombre d'élèves, pour qui les savoirs procéduraux de base en langue orale courante sont acquis, et qui, pourrait-on dire, sont considérés comme ayant déjà culturellement un rapport littéracié à la langue. Nous empruntons cette expression à Bautier (2009, p. 14) qui rappelle pourtant à juste titre que :

Lahire (2008) a[vait] largement étudié les difficultés scolaires des élèves de milieux populaires liées à leur rapport non littéracié à la langue quand les tâches, les exercices, l'acquisition de la grammaire scolaire comme des formes narratives, tout ce qui fait productions scolaires suppose un rapport de familiarité avec cette culture écrite et le statut de langue-objet et non langue de communication qu'elle construit.

10 Dans ce contexte particulier, l'outil grammatical est donc à priori considéré comme efficace pour aider cette population d'élèves à améliorer son rapport à la langue. Nous partageons ce point de vue compte tenu des paramètres d'appropriation linguistique qui sont ceux d'un milieu guidé. Il semble d'ailleurs que même chez les didacticiens qui appuient leurs propositions méthodologiques sur des études neurolinguistiques qui nient la transférabilité des connaissances déclaratives, soit admis à ces dernières au moins un intérêt correctif et mélioratif. Ainsi Germain et Netten fondent-ils leurs propositions d'approche neurolinguistique (ANL) sur des principes issus des travaux neurolinguistiques de Paradis (1994, 2004 \& 2009) selon lesquels il n'y a pas de lien entre la compétence communicative implicite (ou grammaire interne) et le savoir déclaratif. Ce dernier (appelé grammaire externe) est cependant considéré comme utile pour obtenir une communication écrite correcte :

La compétence implicite est nécessaire pour pouvoir communiquer oralement; le savoir explicite est nécessaire pour pouvoir communiquer avec précision en utilisant les formes écrites de la langue. Chaque entité constitue une composante indépendante, mais non suffisante, de l'habileté à utiliser une langue à des fins de communication. Dans la perspective neurolinguistique de l'apprentissage d'une L2/ LÉ, l'équation devient celle-ci : compétence implicite + savoir explicite = habileté à communiquer. L'idée que les deux composantes doivent être développées pour atteindre l'habileté à communiquer constitue un ingrédient essentiel de l'élaboration de l'ANL. (Germain \& Netten, 2012, p. 5)

11 Il nous semble que c'est tout de même admettre l'existence d'un transfert, au moins minimal, entre ces deux « grammaires ». Dans le même esprit, on notera que les théories cognitivistes modernes privilégient le savoir procédural sur le savoir déclaratif, mais n'interdisent pas un recours au déclaratif lorsqu'il s'agit de structurer la mémoire à long terme, celle-ci étant stimulée par des activités de traitement des connaissances qui la 
mettent en rapport avec la mémoire à court terme. S'il n'est donc pas question de trancher la question de savoir si les savoirs explicites se transforment en compétences implicites ou non, il semble au moins raisonnable de penser que dans certaines conditions, celles d'un apprentissage guidé, soumis à des restrictions de temps, de lieu et de multicanalité des savoirs, les savoirs explicites contribuent à l'étayage de la compétence de communication.

Cependant, des facteurs autres que neurologiques ou psychologiques obèrent sans doute la rentabilité de cet étayage. On pense par exemple à une certaine surutilisation de la grammaire dans les cours de FLE ou de FLS, comme l'a montré la recherche CECA ${ }^{3}$ (Carette, Carton \& Vlad, 2011 ; Chnane-Davin, Félix \& Roubaud, 2011), et ce même quand les enseignements sont soi-disant communicatifs. On pense aussi à la faiblesse des descriptions grammaticales proposées, dues au conservatisme déjà évoqué des responsables des politiques éducatives, mais aussi des familles et même de la majorité des enseignants, qui continuent à accorder leur confiance dans les apports de la "grammaire traditionnelle ", au détriment d'apports de connaissances plus récentes de la linguistique (Cuq, 2002). On pense encore au fait que le discours du maitre n'est pas toujours l'adjuvant qu'on pourrait attendre de lui à la compréhension des notions grammaticales proposées aux élèves (Chnane-Davin \& Cuq, $2008 ;$ Ruggia \& Cuq, 2008). On pense enfin à la confusion entre enseignement de la grammaire (enseignement d'un savoir déclaratif sur la langue) et enseignement grammaticalisé de la langue, que nous avons proposé depuis plus de vingt ans déjà (Cuq, 1996), en décrivant la grammaire comme un concept d'ordre didactique plus que comme un concept d'ordre linguistique.

13 Mais surtout peut-être, il faut se souvenir que par ses origines mêmes, la grammaire est inséparable de ce qu'on peut appeler culture de l'écrit. Certains élèves y sont bien sûr déjà entrés, à des degrés divers. Mais il s'agit ici de la culture de l'écrit scolaire, elle-même conçue par l'école comme une propédeutique à la culture de l'écrit social sous toutes ses formes, et notamment celui de la culture cultivée de l'écrit, qu'on opposera à ce que Michel Dabène appelait les écrits (extra)ordinaires (Dabène, 1990) dans le numéro 3 de Lidil. Dans l'article d'ouverture du même numéro, Michel Dabène rappelle que «l'écriture n'est pas l'exercice naturel du langage »(p.17). Cela justifie à nos yeux, pour l'acquérir, l'utilisation du moyen non naturel qu'est la grammaire (au sens courant du terme), encore que la fonction métalinguistique ait été, comme on sait, comptée au nombre des fonctions fondamentales du langage par Jakobson. Mais Dabène posait surtout, dans la continuité de ses travaux de 1987, l'existence d'un "continuum scriptural», ce continuum ayant pour invariant l'activité d'écriture elle-même (p. 16). Et c'est justement l'existence de ce continuum scriptural qui autorise à considérer l'activité d'écriture scolaire comme une propédeutique aux écrits sociaux.

\section{Entrée dans la littéracie chez les élèves allophones scolarisés au collège}

14 Nous aborderons dans cette partie la littéracie en tant que pratique de la lecture (compréhension de l'écrit) et d'écriture (production de l'écrit). Depuis plusieurs décennies le terme d'entrée dans l'écrit a remplacé ce qu'on désignait par l'apprentissage de la lecture et de l'écriture (Le Ferrec, 2008). Si cette entrée s'effectue dans certains milieux avant d'arriver à l'école, chez certains élèves dont le français n'est pas langue 
maternelle, il faut attendre l'arrivée à l'école pour que ce type d'acculturation ait lieu. Dans le cas d'un élève allophone nouvellement arrivé(EANA), cette entrée dans la littéracie au sens de savoir lire et écrire dans une langue seconde est urgente. «Qu'on le veuille ou non+ on doit rentrer dans l'écrit+ parce que c'est la seule porte d'entrée pour suivre++ toutes les évaluations se font par écrit+", dit une enseignante d'EANA ${ }^{4}$. Autrement dit, il faut que l'élève acquière rapidement des compétences minimales de compréhension et de production écrites et les développe de façon à être capable de restructurer sa pensée par la restructuration de son discours dans la langue seconde. L'école ne se contente pas de la littéracie fonctionnelle (Le Ferrec, 2008), mais impose une norme et un niveau d'exigence de littéracie élaborée (nous soulignons) pour paraphraser l'expression « langage élaboré » de Bernstein (1975). Les outils grammaticaux constituent le socle en termes de savoir et de savoir-faire que les élèves allophones, venant d'une autre culture grammaticale et métalinguistique, sont amenés à s'approprier pour s'intégrer dans un système marqué par une tradition grammaticale (Chervel, 1977) où il faut se conformer à la norme. Mais cela peut prendre du temps lorsqu'il s'agit de l'écrit dans une langue étrangère. On ne peut que partager l'interrogation de Vygotski (1997): «Pourquoi le langage écrit est-il difficilement maîtrisé par l'écolier et moins développé chez lui que le langage oral au point que la différence d'âge linguistique entre les deux aspects du langage peut être de six à huit ans à certains stades de l'apprentissage scolaire? » (p. 337) C'est d'autant plus vrai pour les élèves allophones qui se retrouvent dans une double difficulté : entrer dans la littéracie dans leur langue maternelle et reconvertir leurs connaissances pour entrer dans la littéracie dans une langue seconde. Face à ce type de public, des enseignants, souvent peu formés, inventent des façons de faire pour surmonter ces difficultés et amener les élèves à entrer dans la lecture et dans l'écriture en langue seconde. On fait l'hypothèse que plus les élèves maitrisent les outils de la langue française et leur fonctionnalité dans l'évolution d'un texte, plus ils vont comprendre et produire de l'écrit en prenant appui sur des interactions langagières qui vont activer les acquis grammaticaux.

\subsection{Littéracie et tâche de lecture : la compréhension via la grammaire}

Notre analyse s'inscrit dans le cadre restreint de la lecture dans le cadre scolaire où elle demeure la clé pour entrer dans le langage écrit. C'est une activité qui permet en langue seconde (comme d'ailleurs en langue maternelle) d'entrer dans la culture littéraciée. Lors de cette activité qui vise la compréhension d'un énoncé, les enseignants s'appuient sur des stratégies d'approche globale, de repérage d'indices, d'identification des typologies textuelles, de la manipulation du dictionnaire, etc. Comme l'explique Gérard Vigner (2000), un texte qui est écrit dans une LE ou L2 est un texte qui est écrit avec les mots d'une autre langue, mis en succession selon une syntaxe nouvelle. Si elle n'est pas encore maitrisée par les élèves, cette syntaxe peut représenter un obstacle à l'entrée dans la compréhension du déroulement d'un récit. C'est le cas, par exemple, des textes littéraires qui sont souvent utilisés au collège. En effet, entrer dans la compréhension suppose d'être capable de faire un bon usage des outils de la langue, donc de les repérer et de comprendre leur rôle dans le texte. Pour illustrer ce propos, nous présentons un corpus issu d'une séance de classe filmée et transcrite. L'enseignant observé travaille avec des élèves dans une classe de $4^{e}$ dispositif d'accueil et d'intégration (DAI $)^{5}$. Il fait une leçon 
d'étude de texte (lecture analytique) à partir d'un texte de Mérimée, tiré de Matteo Falcone . Au lieu d'entrer par une étude littéraire proprement dite lors de l'analyse du texte, il a fait le choix d'entrer par la grammaire pour amener les élèves à construire le sens. Il se sert du repérage des types de phrase déclarative, interrogative, exclamative et injonctive pour amener les élèves à comprendre l'évolution de l'histoire.

$\mathrm{P}$ : Vous allez faire un travail sur les types de phrase pour commencer. Qu'est-ce qu'on appelle les types de phrase? Rappelez-moi, on avait vu un petit peu au début de l'année?

E : Interrogative...

$\mathrm{P}:$ Rappelez-moi les types de phrase que vous connaissez.

E : Exclamative...

$\mathrm{P}:$ La phrase interrogative.

E : Déclarative...

$\mathrm{P}:$ Exclamative, interrogative et injonctive.

On est dans une phase du réinvestissement des apprentissages qui va servir à construire du sens. Dans l'étude de l'extrait du texte de Mérimée, l'enseignant observé s'appuie particulièrement sur le type injonctif pour mettre l'accent sur un élément essentiel: le moment où le personnage du bandit passe d'une demande d'un service (de le cacher) à une menace de mort.

$\mathrm{P}$ : Euh, j'aimerais voir notamment les phrases injonctives. Sarah, tu vas me les décrire, est-ce que tu les as repérées?

Sarah : Cache-moi.

$\mathrm{P}$ : Alors, la première effectivement c'est dans la première réplique [...] cache-moi

[...]. Alors ensuite c'est?

Sarah : Cache-moi vite.

$\mathrm{P}$ : Alors ensuite c'est cache-moi vite.

Sarah : Cache-moi ou je te tue.

$P$ : Cache-moi ou je te tue.

Dans une situation d'interactions orales de co-construction de la compréhension d'un texte le travail sur la langue, ici la phrase injonctive, on donne du sens aux propos et on entraine une réflexion sur la lisibilité du texte car la syntaxe joue un rôle prépondérant dans le déroulement du récit. Cette démarche d'analyse par un jeu de questions-réponses permet aux élèves d'activer progressivement leurs connaissances grammaticales (déclaratives, procédurales) et de les transférer dans l'activité de lecture. La compétence linguistique développe une compétence en littéracie et contribue à atteindre les objectifs de la séance : étudier et comprendre un texte littéraire. Cette aptitude à comprendre via les outils de la langue permet de traiter l'information. L'enseignant profite à ce stade de l'intérêt que manifestent les élèves pour les trois façons de dire «cache-moi » pour les pousser au-delà de la compréhension littérale :

$\mathrm{P}$ : Samir, il lui donne pas un ordre là. Il lui demande quoi tout simplement? Bon alors à un moment, on pourrait dire, il lui demande un service là. Alors là est-ce qu'il crie ? Non, il lui demande un service. Montrez-moi que le deuxième cache-moi est déjà plus pressé. Qu'est-ce qu'il lui dit ? Cache-moi vite.

$E$ : Là il n'a plus de patience.

$\mathrm{P}$ : Il est déjà un peu plus impatient. D'accord, et alors ça finit par quelle... ? Bon carrément c'est cache-moi ou je te tue. Et là la violence arrive dans les propos du bandit [...] Effectivement il y a, comment on appelle ça, ou je te tue ? Qu'est-ce qu'il fait là ? La menace, une menace grave, une menace de mort.

Après un travail sur trois types de phrases (exclamative, interrogative, injonctive) les plus présents dans le récit dialogué, l'enseignant amène les élèves vers une synthèse sur le 
rôle de chaque type dans la lecture et la compréhension d'un texte où on a inséré un dialogue :

$\mathrm{P}:$ À travers ces types de phrases on voit bien comment évolue le dialogue et on voit, notamment, les sentiments des personnages.

$\mathrm{E}:$ On voit comment les personnages sont présentés.

$\mathrm{P}$ : Pourquoi les trois types de phrases sont les plus présents dans le dialogue? Parce que ça donne beaucoup de plus de vie à un dialogue. Quand vous lisez ce dialogue, vous devez respecter la ponctuation de ces types de phrases.

$\mathrm{E}:$ La phrase exclamative le ton monte.

$\mathrm{P}:$ Vous savez que chaque phrase ne doit pas être prononcée de la même manière

$\mathrm{E}:$ Pas la même musique.

$P$ : Voilà : le désespoir est une chose et la colère c'est autre chose. Quand vous allez lire un dialogue dans un texte avec tous ces types de phrases, il faut à chaque fois adapter le ton de sa voix. Et le ton va exprimer le sentiment. On va faire une relecture à la fin, la plus expressive possible. Maintenant vous allez repérer, on a dit au début que le bandit est en position de force, Fortunato l'enfant est en position de faiblesse. J'aimerais bien que vous me trouviez à quel moment il y a ce qu'on appelle, renversement de situation.

$\mathrm{E}:$ Moi je sais.

$\mathrm{P}:$ Sarah tu attends, tu vas dix fois plus vite que les autres. Allez, on cherche dans le dialogue à quel moment il $\mathrm{y}$ a un changement de position. Vous marquez deux ou trois endroits.

$\mathrm{E}:$ Ton fusil est déchargé.

$\mathrm{P}$ : Non, tu n'as pas compris ma question, je vais la reformuler, à quel moment le bandit est en train de perdre l'influence qu'il croyait avoir sur Fortunato ?

La démarche de compréhension mise en place par l'enseignant fait sortir l'élève du cours classique de grammaire pour le mener vers une compréhension réflexive ou responsive d'une situation où "l'utilisation de la langue s'effectue sous forme d'énoncés concrets " (Bakhtine, 1984, p. 265). La manipulation des mots et de leur fonction n'est plus un exercice théorique mais se déroule selon une attitude responsive-active dans une interaction verbale. Grâce à ces actes de paroles structurés selon le type de chaque phrase dans le récit, l'élève comprend le rôle que jouent ces constructions morphosyntaxiques dans l'évolution du récit, allant du service demandé par le bandit à la menace de mort (type injonctif). Comme l'explique Daniel Faita (2001):

L'énoncé ne surgit de sa texture inerte que si l'interlocuteur donne vie et figure à ces rapports dialogiques multiples qui ne peuvent préexister à l'activité, car la langue en tant que telle, les «propositions » construites par elle, n'en comportent aucune. (p. 20)

Peut-on valider l'hypothèse que les élèves sont bien entrés dans la compréhension du texte via les outils linguistiques issus des types de phrases? Nous pensons que oui si on se base sur les interactions verbales qui ont eu lieu par les réponses des élèves mais aussi par leurs questions, dont l'une est revenue plusieurs fois (c'est quoi l'injonctif?).

\subsection{Littéracie et tâche d'écriture : réinvestir les apprentissages grammaticaux}

21 Les extraits de corpus qui seront évoqués dans cette partie sont issus de productions écrites recueillies sur deux ans de suivi de 15 élèves allophones $\left(6^{e}\right.$ et $\left.5^{e}{ }^{D A I} I^{6}\right)$ lors d'une recherche longitudinale (Chnane-Davin, 2008). Pour analyser ces textes, nous avons retenu le critère principal de cohésion textuelle définie comme «l'ensemble des moyens 
linguistiques qui assurent les liens intra- et interphrastiques permettant à un énoncé oral ou écrit d'apparaitre comme un texte »(Charaudeau \& Maingueneau, 2002, p. 99).

\subsubsection{Des écrits d'élèves allophones au début de leur scolarisation}

À leur arrivée dans le système scolaire français, les élèves observés avaient beaucoup de difficultés à réaliser des productions écrites cohérentes en langue seconde. La tâche d'écriture était pour eux tellement complexe que certains élèves ne savaient pas comment donner du sens à leurs écrits. Il leur manquait des compétences linguistiques, tant lexicales que syntaxiques ou orthographiques. Par exemple, ils avaient une grande difficulté à se situer à la fois dans le temps et dans l'espace (l'antériorité : *J'ai resté, *J'ai viens). Certains ne comprenaient pas le rôle des auxiliaires qui permettent de former le passé composé. Ils associaient, par exemple, l'auxiliaire avoir au présent du verbe venir dont ils ne connaissaient pas encore le participe passé. Ils utilisaient le même procédé pour le verbe rester « *j'ai reste » que pour venir.

Les exemples ci-dessous montrent la grande difficulté de certains élèves.

\section{Exemple 1. - Texte de Lh. en $6^{\mathrm{e}}$ DAI.}

Un soir j'ai tes avec mamer et ma seaur on et ai ou manage pour joue ou mange un momo ils ont quatre filles et trois graçon ils sont à cotes du manage et après ils sont fais du climanger pour des sace du femme et les fille le climange aussi mes acouté des enfant et après ils sont parti es les homme ils sont courire darare eut et après des vouleur ils ferapé un homme et aprè les hommes il son attrapè les vouleur la police ils ont veni ils sont attrapè les ils ont parti

L'élève $\mathrm{a}$, conformément à la consigne (rédiger un texte pour raconter un vol à l'arraché), produit un texte. Elle a indiqué les personnages (je, ma mère, ma sœur, le voleur...) ainsi que le lieu (le manège) et le moment (un soir). Le récit progresse, d'un début vers une fin, en présentant divers évènements. Cependant, la segmentation du texte est quasi absente : aucun alinéa, pas de ponctuation forte ni de majuscule. Les substituts sont utilisés parfois de façon incohérente et on ne comprend pas ce qu'ils remplacent. Les « phrases » du texte manquent de structuration et sont grammaticalement inintelligibles. L'accord sujet/ verbe est inexistant. La conjugaison est méconnue : temps verbaux (présent et passé) mélangés et difficulté à se situer dans le temps. Quant aux règles de l'orthographe, elles sont totalement ignorées : le texte est écrit en partie phonétiquement. Ce dernier point représente une tendance assez présente chez les élèves arabophones dont la langue maternelle est graphophonétique. Cela a évidemment des conséquences sur l'orthographe grammaticale et lexicale, d'où l'absence de sens dans certains passages.

\section{Exemple 2. - Texte de F. en $6^{\mathrm{e}}$ DAI.}

La princesse oreure

Il est une fois une princece cet princese Il est tes tres jantille et la soursiler Il est tes jalouse ebain Il est partait cher la princese mes Il ses queche lavait la prince Il est dormit Il est tes content parsque la princese Il va pas se reveit Il va dormir a tout sa vie la princese ces pas des anné mes Il pas reveit mes et le venue cousin qui lume ces le prince Il fait bizou a la princesse apprés Il s'eréveait acsus du prince.

Le texte produit est court et montre que l'élève n'a pas les compétences scripturales nécessaires pour écrire un texte comportant au minimum 20 lignes (ce qui est exigé lors de l'évaluation nationale). Pour ce qui est de l'énonciation, l'élève a écrit un texte en utilisant la même personne du début jusqu'à la fin. Mais comme c'est la même reprise pronominale qui est utilisée à la fois pour le masculin (le prince) et le féminin (la princesse), cela entraine une certaine difficulté dans le décodage du message. On retrouve un seul élément parmi ceux proposés (la princesse). La ponctuation est absente et le texte 
est écrit sans aucune segmentation. Quant à la syntaxe, les phrases ne sont pas correctes grammaticalement. Enfin, l'écrit est pratiquement phonétique et aucune règle d'orthographe n'est appliquée.

\subsubsection{Des écrits d'élèves allophones suite à la mise en place d'un dispositif spécifique}

On l'a dit, dans un système scolaire fondé sur l'écrit, « apprendre à produire de l'écrit à l'école est une nécessité scolaire» (Verdelhan, 2002, p.105) et une urgence qui conditionnent la réussite. Pour les élèves allophones cependant, un dispositif spécifique s'impose pour les aider à entrer dans l'écriture et à structurer leurs textes (Chnane-Davin, 2008, 2011). Le dispositif mis en place par l'enseignant observé (Chnane-Davin, 2008) s'appuie sur trois objectifs: linguistique, communicatif et culturel. Sa mise en place s'étale sur deux ans $\left(6^{e}\right.$ et $\left.5^{\mathrm{e}}\right)$ afin de préparer l'inclusion des élèves dans une classe ordinaire. L'approche en FLS prévoit de travailler les outils fondamentaux de la langue (grammaire, lexique, orthographe, phonétique) pour amener l'élève à connaitre le fonctionnement du système linguistique français et acquérir les compétences à réinvestir dans les productions orales et écrites. La grammaire est le concept didactique central de ce dispositif, en tant qu'« activité heuristique permettant à l'apprenant de se construire une représentation métalinguistique organisée de la langue qu'il étudie " (Cuq, 1996, p. 41). Les manuels de grammaire en FLE et en FLM offrent un grand choix d'outils qu'il conviendrait d'adapter pour qu'ils servent à l'apprentissage en tant que grammaire de la phrase, du texte et du discours. Les élèves allophones ont besoin de comprendre et d'acquérir «le français [qui] est une langue dotée d'un appareil morphologique assez lourd, [qui] s'organise selon une syntaxe où l'organisation des groupes obéit à des règles complexes, où la traduction orthographique de certains choix grammaticaux occupe une place importante » (Vigner, 2004, p. 8).

Suite à ce dispositif didactique, les données recueillies et analysées ont permis de repérer des indices de progression et d'amélioration de la production écrite en langue seconde. La production des textes choisis se situe à un moment où les élèves entrent dans leur deuxième année d'apprentissage du français (pour certains, davantage). La langue de scolarisation, le français, leur devient plus familière, ce qui facilite les interactions et les aide à entrer de plus en plus dans la langue des consignes orales ou écrites, à les comprendre et à produire des textes en respectant les critères de réalisation. Nous allons citer deux exemples.

Consigne : Imaginez et rédigez la fin du texte étudié en lecture (extrait du conte $\mathrm{La}$ Belle et la Bête).

Exemple 1. - Lh., un an après sa scolarisation en France (en $5^{\mathrm{e}}$ ).

La belle est partie, pour rencontrer le monstre. Le monstre quand il a vue la belle, il est tombé fou amoureux d'elle. La belle lui a parler de son père le marchand, le monstre a repondu ce n'est pas grave. La belle a dit et pourquoi vous avez prouvoquez mon père. Le monstre a dit c'est juste, pour lui faire peur. À la fin de cette histoire, le monstre et la belle ce sont marie. Ils etaient très ameureux entre les deux.

Exemple 2. - F., un an après sa scolarisation en France (en $5^{\mathrm{e}}$ ).

La plus belle fille est partie chez le monstre. Le monstre il aimait boucoup la belle. Alors la bete a proposer a la belle de se marier avec lui. La fille était obligé de faire le truck qui a demandet le monstre. Ils se sont mariée et a chaque fois son père venait la voir. Mai le monstre a changé, il est devenu gentil. Un jour, la bete sauvage 
est devenu un prince beau et charmand. Aprés la fille comme elle a vue, elle était amoureuse de lui et ils étaient trés content.

grammaticalement peut arriver à mieux gérer l'écrit, où « la production du texte oblige l'auteur à gérer une double contrainte : d'une part, introduire des informations nouvelles, d'autre part, assurer la mise en relation de ces dernières avec celles qui étaient déjà disponibles » (Fayol, 1997, p. 218). Autrement dit, réussir à mettre en œuvre un ensemble de processus, ou une «multiplicité de processus » comme le mentionne Gaonac'h (1991), par le transfert d'apprentissage et par une prise de conscience de l'importance de ce transfert pour aboutir à « la fossilisation des compétences ». Pour y arriver, l'élève a besoin d'aides, de «béquilles», tout au moins au début, en s'appuyant sur des textes sources (Chnane-Davin, 2011) étudiés en lecture, qui lui permettent de repérer le rôle du fonctionnement de la langue dans la structuration d'un texte et de respecter la position énonciative. La tâche d'écriture devient, de ce fait, l'acquisition d'une technique consacrée à une forme du discours qui est la narration. À cette fin, les élèves procèdent à une articulation lecture-écriture qui va jusqu'à l'emprunt de mots ou de structures syntaxiques.

L'évolution des écrits de ces élèves est certes lente après deux ans d'étude dans une langue de scolarisation non maternelle et il leur manque encore certains outils de la langue, mais les productions écrites analysées montrent que l'élève passe par plusieurs opérations cognitives : il y a ce que l'élève pense en langue maternelle, ce qu'il traduit en langue seconde (LS), ce qu'il veut écrire en LS, ce qu'il sait écrire en LS et ce qu'il écrit vraiment. 


\section{Conclusion}

30

Atteindre le niveau d'exigence qui est demandé en lecture et en écriture aux élèves des écoles françaises est une tâche ardue pour les élèves allophones comme pour tous ceux qui n'ont pas un accès culturel bien établi à la littéracie. L'école française, imprégnée de grammaire, table sur le fait que l'appropriation d'outils grammaticaux facilite cet accès. Les exemples que nous avons présentés montrent que, globalement, elle n'a pas tort. Pour beaucoup d'élèves cependant, l'acquisition d'un savoir-faire textuel, en compréhension comme en expression, est un processus complexe, aux résultats incertains, parfois très incomplets, et en tout cas plus lent que ne voudrait l'institution. Pour optimiser l'entrée des élèves dans la littéracie, leurs enseignants doivent, de leur côté, posséder des savoirs et un savoir-faire professionnel indispensables: une connaissance ferme de ces outils grammaticaux et des techniques de leur mise en œuvre.

\section{BIBLIOGRAPHIE}

BAKHTINE, Mikhail. (1984). Esthétique de la création verbale (A. Aucouturier, trad.). Paris : Gallimard. BAUTIER, Élisabeth. (2009). Quand le discours pédagogique entrave la construction des usages littéraciés du langage. Pratiques, 143-144. <http://dx.doi.org/10.4000/pratiques.1378> (consulté le 23 avril 2017).

BERNSTEIN, Basil. (1975). Langage et classes sociales. Codes sociolinguistiques et contrôle social. Paris : Minuit.

CARETTE, Emmanuelle, CARTON, Francis \& VLAD, Monica (dir.). (2011). Diversités culturelles et enseignement du français dans le monde : le projet CECA. Grenoble : PUG.

Charaudeau, Patrick \& MAIngueneau, Dominique. (2002). Dictionnaire d'analyse du discours. Paris : Seuil.

CHERVEL, André. (1977). Histoire de la grammaire scolaire. Et il fallut apprendre à écrire à tous les petits Français. Paris : Payot.

CHISs, Jean-Louis. (2008). Littératie et didactique de la culture écrite. Pratiques, 137-138. <http:// dx.doi.org/10.4000/pratiques.1158> (consulté le 24 avril 2017).

CHNANE-DAVIN, Fatima. (2001). La didactique du français langue seconde, continuité ou rupture entre le FLE et le FLM ? (Mémoire de DEA non publié). Aix-Marseille Université, Marseille.

CHNANE-DAVIN, Fatima. (2008 [2005]). Didactique du français langue seconde en France. Le cas de la discipline «français » (Thèse de doctorat, Aix-Marseille Université, Marseille). Lille : Diffusion ANRT.

ChnANe-Davin, Fatima. (2011). Les textes sources pour écrire en FLS. Les Langues modernes, 2, 41-48. 
CHNANE-DAvin, Fatima \& CUQ, Jean-Pierre (dir.). (2008). Du discours de l'enseignant aux pratiques de l'apprenant. Recherches et Applications, 44.

CHNANE-DAVIN, Fatima, FÉLIX, Christine \& RoubAud, Marie-Noëlle. (2011). Le français langue seconde en milieu scolaire français : le projet CECA en France. Grenoble : PUG.

CUQ, Jean-Pierre. (1996). Une Introduction à la didactique de la grammaire en français langue étrangère. Paris : Didier-Hatier.

CUQ, Jean-Pierre. (2002). Du discours du linguiste au discours pédagogique : tribulations de quelques concepts grammaticaux dans les grammaires de référence et les grammaires d'apprentissage. Études de linguistique appliquée, 125, 83-95.

CUQ, Jean-Pierre. (2014). Temps, espace et savoir en didactique du français langue étrangère. Intercambio, 7. Disponible en ligne sur <http://ler.letras.up.pt/site/default.aspx? qry=id05id1184id2686\&sum=sim> (consulté le 4 mars 2017).

DABÈNE, Michel (dir.). (1990). Des écrits (extra)ordinaires. Lidil, 3.

DABÈnE, Michel. (1987). L'adulte et l'écriture. Contribution à une didactique de l'écrit en langue maternelle. Bruxelles : De Boeck / Paris : Éditions universitaires.

FAITA, Daniel. (2001). L'analyse du travail et le statut de « l'activité » chez Bakhtine. Travailler, 6, 13-30.

FAYOL, Michel. (1997). Des idées au texte : psychologie cognitive de la production verbale, orale et écrite. Paris : Presses universitaires de France.

GAONAC'H, Daniel. (1991). Théories d'apprentissage et acquisition d'une langue étrangère. Paris : DidierHatier.

Germain, Claude \& NetTen, Joan. (2012). A New Paradigm for the Learning of a Second or Foreign Language: The Neurolinguistic Approach. Neuroeducation, 1(1), 85-114.

LE FERREC, Laurence. (2008). Littéracie, relations à la culture scolaire et didactique de la lectureécriture en français langue seconde. Dans J.-L. Chiss (dir.), Immigration, école et didactique du français (p. 101-145). Paris : Didier.

MinistÈRE DE l'ÉdUCATION NATIONALE. (2015). Socle commun de compétences, de connaissances et de culture. Bulletin officiel $\mathrm{n}^{\circ} 17 \mathrm{du} 23$ avril 2015. Disponible en ligne sur <www.education.gouv.fr/ pid25535/bulletin_officiel.html?cid_bo=87834> (consulté le 17 avril 2017).

PARADIS, Michel. (1994). Neurolinguistic Aspects of Implicit and Explicit Memory: Implications for Bilingualism. Dans N. Ellis (dir.), Implicit and Explicit Learning of Second Languages (p. 393-419). Londres : Academic Press.

PARADIS, Michel. (2004). A Neurolinguistic Theory of Bilingualism. Amsterdam, Philadelphie : John Benjamins.

PARADIS, Michel. (2009). Declarative and Procedural Determinants of Second Languages. Amsterdam, Philadelphie : John Benjamins.

RUGGIA, Simona \& CUQ, Jean-Pierre. (2008). Le métalangage grammatical en classe de français langue étrangère. Recherches et Applications, 44, 60-69.

VeRDELHAN-BOURGADE, Michèle. (2002). Le français de scolarisation, pour une didactique réaliste. Paris : PUF. 
VÉRONIQUE, Daniel (dir.). (2009). L'acquisition de la grammaire en français langue étrangère. Paris :

Didier.

VIGNER, Gérard. (2000). Enseigner le français comme langue seconde. Paris : Clé International.

VIGNER, Gérard. (2004). La grammaire en FLE. Paris : Hachette.

VyGotski, Lev. (1997 [1985]). Pensée et langage. Paris : La Dispute / La Pensée Sauvage.

\section{NOTES}

1. La plus grande partie des élèves relève du FLS ; les autres sont des élèves en difficulté.

2. Réseau d'éducation prioritaire.

3. L'enseignement du français dans le monde, Culture d'enseignement, culture d'apprentissage (CECA), recherche FIPF/AUP, 2007-2011.

4. Chnane-Davin (2001), extrait d'entretien avec une enseignante de classe d'accueil dans un collège à Marseille.

5. Désigné depuis la circulaire de 2012 par Unité pédagogique pour allophones arrivants (UPE2A).

6. Dispositif d'accueil et d'intégration.

\section{RÉSUMÉS}

Cet article traite de l'entrée en littéracie des élèves allophones nouvellement arrivés en France. À titre préliminaire, nous discuterons brièvement des liens entre les savoirs déclaratifs grammaticaux et les compétences. Par la suite, nous examinerons l'utilisation, par ces élèves, de la grammaire dans l'acquisition des compétences à l'écrit, exigées par le socle commun de connaissances et de compétences. Pour ce faire, nous analyserons deux extraits de corpus de classes, dont le premier montre un travail relatif à la compréhension et le second s'oriente davantage vers un travail sur la production.

This paper is about achieving literacy for allophone pupils recently arrived in France. Preliminarily, we will briefly discuss the relationship between grammatical declarative knowledge and skills. Then, we will focus on the use of grammar by these pupils when acquiring the required common basis of knowledge and skills. To do so, we will analyze two extracts of class records, of which, the first one is a comprehension-related work, while the second is production-based.

\section{INDEX}

Mots-clés : grammaire, littéracie, élèves allophones, compréhension, expression

Keywords : grammar, literacy, allophone pupils, comprehension, production 


\section{AUTEURS}

\section{FATIMA CHNANE-DAVIN}

Aix-Marseille Université, EA 4671 ADEF

\section{JEAN-PIERRE CUQ}

Université Nice Sophia Antipolis, EA 4671 ADEF 